\title{
Synthesis of substituted flavones and chromones using a Wells-Dawson heteropolyacid as catalyst
}

\author{
Daniel O. Bennardi, ${ }^{\text {a,b* }}$ Gustavo P. Romanelli, ${ }^{\text {a,b }}$ Jorge L. Jios, ${ }^{\text {c Juan C. Autino, }}$ \\ Graciela T. Baronetti, ${ }^{\text {d }}$ and Horacio J. Thomas ${ }^{\mathrm{a}}$
}

${ }^{a}$ Centro de Investigación y Desarrollo en Ciencias Aplicadas “Dr. J. J. Ronco" (CINDECA), Departamento de Química, Facultad de Ciencias Exactas, UNLP-CONICET. Calle $47 N^{\circ} 257$, B1900AJK La Plata, Argentina

${ }^{b}$ Cátedra de Química Orgánica, Facultad de Ciencias Agrarias y Forestales, UNLP. Calles 60 y 119, B1904AAN La Plata, Argentina

${ }^{c}$ LaSeISiC, Departamento de Química, Facultad de Ciencias Exactas, UNLP. La Plata, Argentina

${ }^{d}$ Departamento de Ingeniería Química, Facultad de Ingeniería, Universidad de Buenos Aires. Ciudad Universitaria, C1428BG Buenos Aires, Argentina E-mail:gpr@quimica.unlp.edu.ar

\begin{abstract}
In this work, we report the use of bulk and silica-supported Wells-Dawson acid $\left(\mathrm{H}_{2} \mathrm{P} 2 \mathrm{~W}_{18} \mathrm{O}_{62} \cdot 24 \mathrm{H}_{2} \mathrm{O}\right)$ as reusable, heterogeneous catalysts to obtain substituted flavones and chromones for the cyclization of 1-(2-hydroxyphenyl)-3-aryl-1,3-propanediones. The reaction experiments were performed using toluene as solvent at reflux and in the absence of solvent, at $110{ }^{\circ} \mathrm{C}$. Under these conditions eleven examples were obtained with very good yields (82-91\%) and high selectivity. The catalysts were easily recycled and reused without loss of their catalytic activity. The presented synthetic method is a simple, clean and environmentally friendly alternative for synthesizing substituted flavones and chromones.
\end{abstract}

Keywords: $\mathrm{H}_{2} \mathrm{P}_{2} \mathrm{~W}_{18} \mathrm{O}_{62} \cdot 24 \mathrm{H}_{2} \mathrm{O}$, silica gel-supported catalyst, flavones, chromones, acid catalysis

\section{Introduction}

The compounds that contain the chromone skeleton (4H-benzopyran-4-one) (flavones and chromones) are widely spread in nature, and they are part of the flavonoid family. ${ }^{1}$ These compounds have been reported to exhibit multiple biological properties, for example antibacterial, antifungal, ${ }^{2,3}$ anticancer, ${ }^{1}$ antioxidant, ${ }^{4}$ and anti-HIV. ${ }^{5}$ Some flavonoids inhibit the 
histamine release from human basophils and rat mast cells. ${ }^{6}$ Moreover, it is known that some flavonoids have a repelling property against some phytophagous insects and a subterranean termite (Coptotermes sp.) acting as an antifeedant. ${ }^{7,8}$.

The importance of these compounds has led to the development of various methods for their synthesis: for example, the Allan-Robinson strategy, from chalcones and via an intramolecular Wittig strategy. ${ }^{9,10}$ One of the most common methods involves acylation of an ohydroxyacetophenone with an aromatic acid chloride yielding an aryl ester. The ester is then rearranged by a base (the Baker-Venkataraman rearrangement) to a 1,3-diaryl 1,3-diketone. ${ }^{11}$ The latter compound gives a 2-arylchromone on cyclocondensation. This is usually a catalyzed reaction and it has been carried out in different media. Some reaction conditions employed were the use of excess of sulfuric acid in glacial acetic acid, ${ }^{12}$ cationic exchange resins in isopropanol, ${ }^{13}$ glacial acetic acid-anhydrous sodium acetate or aqueous potassium carbonate. ${ }^{14}$ Greener procedures have been recently described, for example using $\mathrm{CuCl}_{2}$ in ethanol, ${ }^{15}$ and ionic liquid under microwave irradiation. ${ }^{16}$

The use of typical acid catalysts, such as sulfuric, phosphoric, hydrofluoric acids, or boron trifluoride, leads to environmental pollution and corrosion problems, ${ }^{17}$ which can be avoided by using an insoluble acid, for example a heteropolyacid (HPA) as catalyst. HPAs are useful solid catalysts because of their superacidic properties; ${ }^{18}$ catalysis by HPAs and related compounds is a field of increasing importance worldwide. Numerous developments are being carried out in basic research as well as in technological processes. ${ }^{19,20,21}$ Varied reactions are currently catalyzed by HPAs, such as dehydration, ${ }^{22}$ cyclization, ${ }^{17,23}$ esterification, ${ }^{24}$ amine oxidation and olefin epoxidation. $^{25}$ HPAs are widely applicable in the production of fine chemicals such as fragrances, pharmaceuticals and food. ${ }^{26}$

We have recently applied the heteropolyacid catalyst $\mathrm{H}_{6} \mathrm{P}_{2} \mathrm{~W}_{18} \mathrm{O}_{62} .24 \mathrm{H}_{2} \mathrm{O}$ with WellsDawson structure to different reactions. Among them, preparations of acylals, ${ }^{27}$ coumarins $^{28}$, and diphenylmethyl ethers ${ }^{29}$ could be mentioned.

In continuation of our work on the catalytic properties of Wells-Dawson heteropolyacids, herein we report the use of bulk and silica-supported Wells-Dawson acid as a reusable, heterogeneous catalyst to obtain substituted flavones and chromones for the cyclization of 1-(2hydroxyphenyl)-3-aryl-1,3-propanediones (Scheme 1).<smiles>[R]c1ccc(O)c(C(=O)CC(=O)[Al])c1</smiles>

1

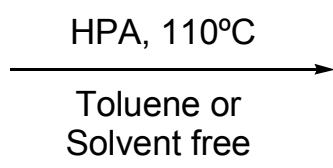

$$
\text { Ar }=\text { Phenyl }
$$

Furyl

1-Naphthyl

2-Naphthyl

\section{Scheme 1}<smiles>[R]c1ccc2oc(Br)cc(=O)c2c1</smiles>

\section{2}




\section{Results and Discussion}

Initially we conducted blank experiments without the presence of Wells-Dawson acid. No product was detected when $1 \mathrm{mmol}$ of 1-(2-hydroxyphenyl)-3-phenyl-1,3-propanedione dissolved in toluene was refluxed for $5 \mathrm{~h}$. When this solution was refluxed in the presence of silica (support), no product was detected by TLC.

Afterwards the catalytic activity of the bulk Wells-Dawson acid (WD) and that of WellsDawson acid containing $40 \%$ by weight of the acid $\left(\mathrm{WD}_{40} / \mathrm{SiO}_{2}\right)$ was tested in the preparation of substituted flavones and chromones. The obtained results are shown in Table 1. The experiments were carried out in refluxing toluene, in the presence of $1 \% \mathrm{mmol}$ support catalyst, and in the absence of solvent (solvent-free), in the presence of $1 \%$ mmol bulk catalyst.

Temperature and molar ratio of the Wells-Dawson acid to substrates were checked to optimize the reaction, using 1-(2-hydroxyphenyl)-3-phenyl-1,3-propanedione as substrate. No reaction was detected at room temperature. The use of just $1 \%$ mmol of HPA is enough to push the reaction forward; higher amounts of the catalyst did not improve the results.

The experiments were run until 1,3-diketone was consumed or until no changes in the composition of the reaction mixture were observed, in both the toluene solution and excluding the solvent. In all the cases, the desired products were obtained with high selectivity, almost free of secondary products. The unchanged starting materials were recovered nearly quantitatively. Recycling of both catalysts (Table 1, entries 1 and 8) was checked in two consecutive batches after the first one; the catalysts showed almost constant activity. The yields obtained using WD in the absence of solvent were similar to those involving $\mathrm{WD}_{40} / \mathrm{SiO}_{2}$ in refluxing toluene. However, the experiments performed in solvent-free conditions showed a substantial reduction of the reaction times. No stereoelectronic effects owing to the substituent were observed on the yield, for each of the catalysts. The yields of flavones were similar to those of chromones (Table 1).

\section{Conclusions}

The method described above provides a clean, simple and useful alternative for preparing substituted flavones and chromones. The use of $\mathrm{WD}$ and $\mathrm{WD}_{40} / \mathrm{SiO}_{2}$ catalysts provides very good yields, also leading to an easy separation and recovering of the catalysts for further use. The catalytic activity, which is practically constant in consecutive reaction batches, and the high recovery of the catalysts allow for both low environmental impact and low cost. Other "green" advantages of the method are the low formation of wastes and the replacement of corrosive, soluble mineral acids. 
Table 1. Preparation of flavones and chromones using $1 \%$ both bulk and silica-supported $\mathrm{H}_{6} \mathrm{P}_{2} \mathrm{~W}_{18} \mathrm{O}_{62} \cdot 24 \mathrm{H}_{2} \mathrm{O}$

\begin{tabular}{|c|c|c|c|c|c|}
\hline Entry & Flavone/Chromone & $\begin{array}{l}\text { Time (h) } \\
(\mathrm{WD})^{\mathrm{a}}\end{array}$ & $\begin{array}{l}\text { Yield (\%) } \\
(\mathrm{WD})^{\mathrm{a}}\end{array}$ & $\begin{array}{c}\text { Time }(\mathrm{h}) \\
\left(\mathrm{WD}_{40} / \mathrm{SiO}_{2}\right)^{\mathrm{b}}\end{array}$ & $\begin{array}{c}\text { Yield }(\%) \\
\left(\mathrm{WD}_{40} / \mathrm{SiO}_{2}\right)^{\mathrm{b}}\end{array}$ \\
\hline 1 & & 0.5 & $87(88,88)^{\mathrm{c}}$ & 4.5 & $91(90,90)^{\mathrm{c}}$ \\
\hline 2 & & 0.7 & 87 & 4.5 & 86 \\
\hline 3 & & 0.5 & 86 & 4.5 & 85 \\
\hline 4 & & 0.5 & 86 & 5 & 86 \\
\hline 5 & & 0.5 & 86 & 5 & 87 \\
\hline 6 & & 0.5 & 82 & 4 & 83 \\
\hline 7 & & 0.6 & 85 & 4.5 & 84 \\
\hline 8 & & 0.7 & $88(86,86)^{\mathrm{c}}$ & 5 & $87(86,85)^{\mathrm{c}}$ \\
\hline 9 & & 0.7 & 88 & 5 & 88 \\
\hline 10 & & 0.8 & 85 & 5 & 87 \\
\hline 11 & & 0.6 & 87 & 4.5 & 87 \\
\hline
\end{tabular}

${ }^{\mathrm{a}}$ Solvent-free and ${ }^{\mathrm{b}}$ in toluene, $110{ }^{\circ} \mathrm{C}$. ${ }^{\mathrm{c}}$ Yields obtained in the first and second reuse of the catalyst. 


\section{Experimental Section}

General Procedures. All the yields were calculated from crystallized products. All the products were identified by comparison of analytical data (mp, TLC, NMR) with those reported or with authentic samples prepared by the conventional method using sulfuric acid as catalyst. All the starting 1,3-diketones were prepared following a procedure described elsewhere. ${ }^{30}$ Melting points of the compounds were determined in open capillary tubes and are uncorrected. ${ }^{13} \mathrm{C}$ NMR and ${ }^{1} \mathrm{H}$ NMR spectra were recorded at room temperature on Bruker AC-250 and Bruker Avance DPX-400 spectrometers using TMS as internal standard.

\section{Catalyst preparations}

The Dawson acid $\left(\mathrm{H}_{6} \mathrm{P}_{2} \mathrm{~W}_{18} \mathrm{O}_{62} \cdot 24 \mathrm{H}_{2} \mathrm{O}\right)$ was prepared by the Drechsel method ${ }^{31}$ from a $\alpha / \beta$ $\mathrm{K}_{6} \mathrm{P}_{2} \mathrm{~W}_{18} \mathrm{O}_{62} .10 \mathrm{H}_{2} \mathrm{O}$ isomer mixture. This Dawson-type salt was prepared according to the technique reported by Lyon et al. ${ }^{32}$ Concentrated $\mathrm{H}_{3} \mathrm{PO}_{4}$ in a 4:1 acid/salt ratio was added to a boiling aqueous solution of $\mathrm{Na}_{2} \mathrm{WO}_{4} \cdot 2 \mathrm{H}_{2} \mathrm{O}$, and the mixture was kept boiling in a reflux system for $8 \mathrm{~h}$. The salt was precipitated by adding $\mathrm{KCl}$, then purified by recrystallization and cooled overnight to $278 \mathrm{~K}$. The product, which is a mixture of the $\alpha$ and $\beta$ isomers, was filtered, washed and then vacuum-dried for $8 \mathrm{~h}$.

The acid was obtained from an aqueous solution of $\alpha / \beta \mathrm{K}_{6} \mathrm{P}_{2} \mathrm{~W}_{18} \mathrm{O}_{62} .10 \mathrm{H}_{2} \mathrm{O}$ salt, which was treated with ether and concentrated $\mathrm{HCl}(37 \%)$ solution. The Dawson acid so released formed an addition compound with the ether, which allows it to be separated from the solution. After obtaining the ether solution with the acid, the ether was eliminated by flowing dry air, and the remaining solution was placed in a vacuum-desiccator until crystallization.

Silica-supported Wells-Dawson acids $\left(\mathrm{WD}_{40} / \mathrm{SiO}_{2}\right)$ were prepared by wet impregnation of Grace Davison silica (Grade 59, specific area $=250 \mathrm{~m}^{2} / \mathrm{g}$ ) with an aqueous solution of the synthesized WD acid. A catalyst containing $40 \mathrm{wt} \%$ of WD was prepared. After impregnation, samples were dried at room temperature in a vacuum-desiccator for $8 \mathrm{~h}$.

\section{General procedures for the preparation of substituted flavones and chromones}

(a) Reaction in toluene solution. A mixture of 1,3-diketone $(0.5 \mathrm{mmol})$ dissolved in $3 \mathrm{ml}$ toluene and $\mathrm{WD}_{40} / \mathrm{SiO}_{2}$ catalyst $(1 \% \mathrm{mmol}$ ) was refluxed with stirring for the indicated time (see Table 1). The reaction was followed by TLC using TLC aluminum sheets (silica gel $60 \mathrm{~F}_{254}$ Merck). When the reaction time was over, the catalyst was filtered and washed twice with toluene $(1 \mathrm{ml})$. The extracts were combined and washed with $3 \mathrm{M} \mathrm{NaOH}$, then with $\mathrm{H}_{2} \mathrm{O}$, and dried with anhydrous sodium sulfate. The organic solution was concentrated in vacuum. All the solid crude products were recrystallized from methanol.

(b) Solvent-free reaction. A mixture of 1,3-diketone $(0.5 \mathrm{mmol})$ and WD catalyst $(1 \% \mathrm{mmol})$ was stirred at $110{ }^{\circ} \mathrm{C}$ for the indicated time (Table 1). The reaction mixture was extracted with hot toluene $(3 \times 2 \mathrm{ml})$. The solution was washed with $\mathrm{NaOH} 3 \mathrm{M}$ and $\mathrm{H}_{2} \mathrm{O}$; then it was dried with 
anhydrous sodium sulfate and concentrated in vacuum. All the solid crude products were recrystallized from methanol.

Flavone. Mp: $97-98{ }^{\circ} \mathrm{C}$ (methanol) (lit. mp: $\left.98{ }^{\circ} \mathrm{C}^{33}\right) ;{ }^{13} \mathrm{C} \mathrm{NMR}\left(100 \mathrm{MHz}, \mathrm{CDCl}_{3}\right) \delta 107.3$, $117.9,123.7,124.9,125.5,126.0,129.0,131.5,131.8,133.5,155.9,163.1,177.9 ;{ }^{1} \mathrm{H}$ NMR $(400$ $\left.\mathrm{MHz}, \mathrm{CDCl}_{3}\right) \delta 6.82(\mathrm{~s}, 1 \mathrm{H}), 7.41(\mathrm{ddd}, 1 \mathrm{H}, \mathrm{J}=1.0,7.2,8.2 \mathrm{~Hz}), 7.49-7.56(\mathrm{~m}, 4 \mathrm{H}), 7.68$ (ddd, $1 \mathrm{H}, \mathrm{J}=1.7,7.2,8.3 \mathrm{~Hz}), 7.92-7.95(\mathrm{~m}, 2 \mathrm{H}), 8.23(\mathrm{dd}, 1 \mathrm{H}, \mathrm{J}=1.8,8.2 \mathrm{~Hz})$.

6-Chloroflavone. $\mathrm{Mp}: 185-186{ }^{\circ} \mathrm{C}$ (methanol) (lit. mp: $\left.183-184{ }^{\circ} \mathrm{C}^{34}\right) ;{ }^{13} \mathrm{C} \mathrm{NMR} \mathrm{(100} \mathrm{MHz,}$ $\left.\mathrm{CDCl}_{3}\right) \delta 107.5,119.9,125.0,125.2,126.3,129.1,131.2,131.4,131.9,134.0,154.5,163.6$, 177.2; ${ }^{1} \mathrm{H}$ NMR $\left(400 \mathrm{MHz}, \mathrm{CDCl}_{3}\right) \delta 6.84(\mathrm{~s}, 1 \mathrm{H}), 7.54-7.56(\mathrm{~m}, 4 \mathrm{H}), 7.65(\mathrm{dd}, 1 \mathrm{H}, \mathrm{J}=2.5,8.8$ $\mathrm{Hz})$, 7.91-7.94 (m, 2H), 8.20 (d, 1H, J=2.6 Hz).

7-Chloroflavone. Mp: $159-160{ }^{\circ} \mathrm{C}$ (methanol) (lit. mp: $\left.156-157{ }^{\circ} \mathrm{C}^{35}\right) ;{ }^{13} \mathrm{C} \mathrm{NMR}(62.5 \mathrm{MHz}$, $\left.\mathrm{CDCl}_{3}\right) \delta 107.2,118.2,122.4,126.0,126.3,126.8,129.0,131.3,131.7,139.6,156.0,163.2$, 177.9; ${ }^{1} \mathrm{H}$ NMR $\left(250 \mathrm{MHz}, \mathrm{CDCl}_{3}\right) \delta 6.82(\mathrm{~s}, 1 \mathrm{H}), 7.42(\mathrm{dd}, 1 \mathrm{H}, \mathrm{J}=1.9,8.4 \mathrm{~Hz}), 7.52-7.55(\mathrm{~m}$, $3 \mathrm{H}), 7.61(\mathrm{~d}, 1 \mathrm{H}, \mathrm{J}=1.9 \mathrm{~Hz}), 7.90-7.93(\mathrm{~m}, 2 \mathrm{H}), 8.22(\mathrm{~d}, 1 \mathrm{H}, \mathrm{J}=8.4 \mathrm{~Hz})$.

6-Methylflavone. Mp: $120-121{ }^{\circ} \mathrm{C}$ (methanol) (lit. mp: $\left.122-123{ }^{\circ} \mathrm{C}^{36}\right) ;{ }^{13} \mathrm{C} \mathrm{NMR} \mathrm{(100} \mathrm{MHz,}$ $\left.\mathrm{CDCl}_{3}\right) \delta 20.9,107.3,117.7,123.5,124.9,126.2,128.9,131.4,131.8,134.9,135.1,154.4,163.1$, 178.5; ${ }^{1} \mathrm{H}$ NMR $\left(400 \mathrm{MHz}, \mathrm{CDCl}_{3}\right) \delta 2.47(\mathrm{~s}, 3 \mathrm{H}), 6.82(\mathrm{~s}, 1 \mathrm{H}), 7.46-7.54(\mathrm{~m}, 5 \mathrm{H}), 7.92-7.94(\mathrm{~m}$, $2 \mathrm{H}), 8.02(\mathrm{~s}, 1 \mathrm{H})$.

7-Methylflavone. Mp: $122-124{ }^{\circ} \mathrm{C}$ (methanol) (lit. p.f.: $\left.120{ }^{\circ} \mathrm{C}^{37}\right) ;{ }^{13} \mathrm{C}$ NMR $\left(62.5 \mathrm{MHz}, \mathrm{CDCl}_{3}\right)$ $\delta 22.0,107.5,118.0,122.0,126.0,126.8,127.2,129.0,131.7,132.0,145.1,156.3,163.2,178.2$; ${ }^{1} \mathrm{H}$ NMR $\left(250 \mathrm{MHz}, \mathrm{CDCl}_{3}\right) \delta 2.45(\mathrm{~s}, 3 \mathrm{H}), 6.75(\mathrm{~s}, 1 \mathrm{H}), 7.20(\mathrm{~d}, 1 \mathrm{H}, \mathrm{J}=9.9 \mathrm{~Hz}), 7.30(\mathrm{~s}, 1 \mathrm{H})$, 7.40-7.55 (m, 3H), 7.91-7.97 (m, 2H), 8.10 (d, 1H, J=9.9 Hz).

7-Methoxyflavone. Mp: $109-110{ }^{\circ} \mathrm{C}$ (methanol) (lit. mp: $\left.110{ }^{\circ} \mathrm{C}^{38}\right) ;{ }^{13} \mathrm{C} \mathrm{NMR}(100 \mathrm{MHz}$, DMSO-d $\left._{6}\right) \delta 56.3,101.1,106.9,114.9,117.3,126.3,126.4,129.3,131.3,131.8,157.7,162.3$, 164.1, 176.6; ${ }^{1} \mathrm{H}$ NMR (400 MHz, DMSO-d 6 ) $\delta 3.92$ (s, 3H), 6.97 (s, 1H), 7.07 (dd, 1H, J= 2.4, $8.8 \mathrm{~Hz}), 7.32(\mathrm{~d}, 1 \mathrm{H}, \mathrm{J}=2.4 \mathrm{~Hz}), 7.58-7.60(\mathrm{~m}, 3 \mathrm{H}), 7.94(\mathrm{~d}, 1 \mathrm{H}, \mathrm{J}=8.8 \mathrm{~Hz}), 8.05-8.12(\mathrm{~m}, 2 \mathrm{H})$.

6-Bromoflavone. Mp: 190-191 ${ }^{\circ} \mathrm{C}$ (methanol) (lit. mp: $\left.189-190{ }^{\circ} \mathrm{C}^{39}\right) ;{ }^{13} \mathrm{C} \mathrm{NMR}(100 \mathrm{MHz}$, $\left.\mathrm{CDCl}_{3}\right) \delta 107.5,119.5,119.9,125.1,126.3,128.1,129.1,131.1,131.9,136.2,154.6,163.8$, 178.0; ${ }^{1} \mathrm{H}$ NMR $\left(400 \mathrm{MHz}, \mathrm{CDCl}_{3}\right) \delta 6.82(\mathrm{~s}, 1 \mathrm{H}), 7.52-7.55(\mathrm{~m}, 4 \mathrm{H}), 7.83(\mathrm{dd}, 1 \mathrm{H}, \mathrm{J}=2.4,8.3$ $\mathrm{Hz})$, 7.89-7.92 (m, 2H), 8.35 (d, $1 \mathrm{H}, \mathrm{J}=2.4 \mathrm{~Hz})$.

2-(2-Naphthyl)chromone. Mp: $162-163{ }^{\circ} \mathrm{C}$ (methanol) (lit. mp: $\left.164-165{ }^{\circ} \mathrm{C}^{38}\right) ;{ }^{13} \mathrm{C}$ NMR $(100$ $\left.\mathrm{MHz}, \mathrm{CDCl}_{3}\right) \delta 107.9,118.1,122.3,124.0,125.2,125.7,126.7,126.9,127.6,128.0,128.7$, 128.7, 129.1, 132.9, 133.8, 134.5, 156.3, 163.4, 178.3; ${ }^{1} \mathrm{H}$ NMR (400 MHz, $\left.\mathrm{CDCl}_{3}\right) \delta 6.94(\mathrm{~s}$, $1 \mathrm{H}), 7.43(\mathrm{ddd}, 1 \mathrm{H}, \mathrm{J}=1.6,6.6,7.9 \mathrm{~Hz}), 7.54-7.60(\mathrm{~m}, 2 \mathrm{H}), 7.63$ (d, 1H, J=8.0 Hz), 7.72 (ddd, $1 \mathrm{H}, \mathrm{J}=1.6,6.6,7.9 \mathrm{~Hz}), 7.87-8.05(\mathrm{~m}, 4 \mathrm{H}), 8.23(\mathrm{dd}, 1 \mathrm{H}, \mathrm{J}=1.5,7.9 \mathrm{~Hz}), 8.46(\mathrm{~s}, 1 \mathrm{H})$.

7-Methyl-2-(1-naphthyl)chromone. Mp: $162-163{ }^{\circ} \mathrm{C}$ (methanol) (lit. no data); ${ }^{13} \mathrm{C}$ NMR (62.5 $\left.\mathrm{MHz}_{2} \mathrm{CDCl}_{3}\right) \delta 21.8,113.0,118.0,121.9,124.9,125.0,125.6,126.5,126.8,127.3,127.8,128.7$, $130.5,130.9,131.4,133.8,145.2,156.9,165.1,178.1 ;{ }^{1} \mathrm{H} \mathrm{NMR}\left(250 \mathrm{MHz}, \mathrm{CDCl}_{3}\right) \delta 2.50(\mathrm{~s}$, 
$3 \mathrm{H}), 6.65(\mathrm{~s}, 1 \mathrm{H}), 7.27(\mathrm{~d}, 1 \mathrm{H}, \mathrm{J}=9.3 \mathrm{~Hz}), 7.32(\mathrm{~s}, 1 \mathrm{H}), 7.54-7.60(\mathrm{~m}, 3 \mathrm{H}), 7.75(\mathrm{dd}, 1 \mathrm{H}, \mathrm{J}=0.8$, $6.7 \mathrm{~Hz}), 7.92-7.96(\mathrm{~m}, 1 \mathrm{H}), 8.01(\mathrm{~d}, 1 \mathrm{H}, \mathrm{J}=8.3 \mathrm{~Hz}), 8.11-8.15(\mathrm{~m}, 1 \mathrm{H}), 8.18(\mathrm{~d}, 1 \mathrm{H}, \mathrm{J}=8.1 \mathrm{~Hz})$.

7-Chloro-2-(1-naphthyl)chromone. Mp: $198-199{ }^{\circ} \mathrm{C}$ (methanol) (lit. mp: $198-199{ }^{\circ} \mathrm{C}^{23}$ ); ${ }^{13} \mathrm{C}$ NMR $\left(100 \mathrm{MHz}, \mathrm{CDCl}_{3}\right) \delta 113.3,118.3,122.5,124.7,125.1,126.2,126.7,127.2,127.6,128.0$, 128.8, 130.2, 130.3, 131.7, 133.7, 139.9, 156.8, 165.6, 177.4; ${ }^{1} \mathrm{H}$ NMR (400 MHz, $\left.\mathrm{CDCl}_{3}\right) \delta 6.70$ $(\mathrm{s}, 1 \mathrm{H}), 7.46(\mathrm{dd}, 1 \mathrm{H}, \mathrm{J}=1.9,8.5 \mathrm{~Hz}), 7.58-7.63(\mathrm{~m}, 4 \mathrm{H}), 7.78(\mathrm{dd}, 1 \mathrm{H}, \mathrm{J}=1.1,7.2 \mathrm{~Hz}), 7.97-7.99$ $(\mathrm{m}, 1 \mathrm{H}), 8.06(\mathrm{~d}, 1 \mathrm{H}, \mathrm{J}=8.2 \mathrm{~Hz}), 8.12-8.14(\mathrm{~m}, 1 \mathrm{H}), 8.26(\mathrm{~d}, 1 \mathrm{H}, \mathrm{J}=8.6 \mathrm{~Hz})$.

2-(2-Furyl)chromone. Mp: $134-135{ }^{\circ} \mathrm{C}$ (methanol) (lit. mp: $135{ }^{\circ} \mathrm{C}^{40}$ ). ${ }^{13} \mathrm{C} \mathrm{NMR}(62.5 \mathrm{MHz}$, $\left.\mathrm{CDCl}_{3}\right) \delta 105.5,112.4,112.9,117.8,124.2,125.1,125.7,133.6,145.7,146.4,155.1,155.8$, 177.6; ${ }^{1} \mathrm{H}$ NMR $\left(250 \mathrm{MHz}, \mathrm{CDCl}_{3}\right) \delta 6.58-6.60(\mathrm{~m}, 1 \mathrm{H}), 6.71(\mathrm{~s}, 1 \mathrm{H}), 7.11(\mathrm{~d}, 1 \mathrm{H}, \mathrm{J}=3.4 \mathrm{~Hz})$, $7.38(\mathrm{~s}, 1 \mathrm{H}), 7.47$ (d, 1H, J= 8.3 Hz), 7.61-7.69 (m, 2H), $8.19(\mathrm{dd}, 1 \mathrm{H}, \mathrm{J}=1.2,7.5 \mathrm{~Hz})$.

\section{Acknowledgements}

We thank Agencia Nacional de Promoción Científica y Tecnológica (Argentina), Fundación Antorchas, Universidad Nacional de La Plata, and CONICET for financial support.

\section{References}

1. Martens, S.; Mithöfer, A. Phytochemistry 2005, 66, 2399.

2. Alam, S. J. Chem. Sci. 2004, 116, 325.

3. Göker, H.; Boykin, D.; Yildiz, S. Bioorg. Med. Chem. 2005, 13, 1707.

4. Chu, H.; Wu, H.; Lee, Y. Tetrahedron 2004, 60, 2647.

5. Wu, J.; Wang, X.; Yi, Y.; Lee, K. Bioorg. Med. Chem. Lett. 2003, 13, 1813.

6. Yano, S.; Tachibana, H.; Yamada, K. J. Agric. Food Chem. 2005, 53, 1812.

7. Morimoto, M.; Tanimoto, K.; Nakano, S.; Ozaki, T.; Nakano, A.; Komai, K. J. Agric. Food Chem. 2003, 51, 389.

8. Ohmura, W.; Doi, S.; Aoyama, M.; Ohara, S. J. Wood Sci. 2000, 46, 149.

9. Barton, D.; Ollis, W. Comprehensive Organic Chemistry. The Synthesis and Reactions of Organic Compounds, Vol. 4, Pergamon, Oxford, 1979, 659.

10. Ganguly, A.; Kaur, S.; Mahata, P.; Biswas, D.; Pramanik, B.; Chan, T. Tetrahedron Lett. 2005, 46, 4119 and references cited therein.

11. Varma, R.; Saini, R.; Kumar, D. J. Chem. Research (S) 1998, 348 and references cited therein.

12. Wheeler, T. Organic Syntheses, 1952, 32, 72.

13. Hoshino, Y.; Takeno, N. Bull. Chem. Soc. Jpn. 1987, 60, 1919.

14. Saxena, S.; Makrandi, J.; Grover, S. Synthesis 1985, 697.

15. Kabalka, G.; Mereddy, A. Tetrahedron Lett. 2005, 46, 6315. 
16. Sarda, S.; Pathan, M.; Paike, V.; Pachmase, P.; Jadhav, W.; Pawar, R. ARKIVOC 2006, (xvi), 43.

17. Vázquez, P.; Pizzio, L.; Romanelli, G.; Autino, J.; Cáceres, C.; Blanco, M.; Appl. Catal. A: General 2002, 235, 233.

18. Kozhevnikov, I. Chem. Rev. 1998, 98, 171.

19. Misono, M. Korean J. Chem. Eng. 1997, 14, 427.

20. Zhao, Z.; Li, Z.; Wang, G.; Qiao, W.; Cheng, L. Prog. Chem. 2004, 16, 620.

21. Yadav, G. Catal. Surv. Asia 2005, 9, 117.

22. Turek, W.; Haber, J.; Krowiak, A. Appl. Surf. Sci. 2005, 252, 823.

23. Bennardi, D.; Romanelli, G.; Jíos, J.; Vázquez, P.; Cáceres, C.; Autino, J. Heterocyclic Communications 2007, 13, 77.

24. Pizzio, L.; Vázquez, P.; Cáceres, C.; Blanco, M. Appl. Catal. A: General 2003, 256, 125.

25. Ding, Y.; Ma, B.; Gao, Q.; Li, G.; Yan, L.; Suo, J. J. Mol. Catal. A: Chem. 2005, 230, 121.

26. Brunel, D.; Blanc, A.; Galarneau, A.; Fajula, F. Catal. Today 2002, 73, 139.

27. Romanelli, G.; Thomas, H.; Baronetti, G.; Autino, J. Tetrahedron Lett. 2003, 44, 1301.

28. Romanelli, G.; Bennardi, D.; Ruiz, D.; Baronetti, G.; Thomas, H.; Autino, J. Tetrahedron Lett. 2004, 45, 8935.

29. Romanelli, G.; Ruiz, D.; Bideberripe, H.; Autino, J.; Baronetti, G.; Thomas, H. ARKIVOC 2007, (xvii), 1.

30. Jíos, J.; Autino, J.; Pomilio, A. An. Asoc. Quím. Argent. 1995, 83, 183.

31. Baronetti, G.; Briand, L.; Sedran, U.; Thomas, H. Appl. Catal. A: General 1998, 173, 265.

32. Lyon, D.; Miller, W.; Novet, T.; Domaille, P.; Evitt, E.; Johnson, D.; Finke, R. J. Am. Chem. Soc. 1991, 113, 7209.

33. Furniss, B.; Hannaford, A.; Rodgers, V.; Smith, P.; Tatchell, A. Vogel's Textbook of Practical Organic Chemistry; Longman: New York, 1978.

34. Ruhemann, S. Ber. 1921, 54b, 912.

35. Chen, F.; Chang, C. J. Chem. Soc. 1958, 146.

36. Beilstein Handbuch der Organischen Chemie, XVII, I 206, II 400.

37. Robertson, A.; Waters, R.; Jones, E. J. Chem. Soc. 1932, 1681.

38. Virkar, V.; Shah, R. J. Univ. Bombay 1942, 11, 140.

39. Chang, C.; Cheng, F. J. Chem. Soc. 1961, 664, 3414.

40. Ollis, W.; Weight, D. J. Chem. Soc. 1952, 732, 3826. 